\title{
On the way to convergence between general educational courses of informatics and technology
}

\author{
Sergey Beshenkov ${ }^{1,2, *}$, Eteri Mindzaeva ${ }^{2}$, and Sergey Neustroev ${ }^{1}$ \\ ${ }^{1}$ Institute of Management in Education of the Russian Academy of Education, 105062, 5/16, 1B, \\ Makarenko str., Moscow, Russia \\ ${ }^{2}$ Academy of Social Management, 141006, 13 Industrialnaya str., Mytischi, Russia
}

\begin{abstract}
The article concerns the conceptual fundamentals of convergence for general educational courses in informatics and technology. The necessity of such step is determined by the urgency of adequate responses to the challenges of the current information civilization: exponential growth of non-systematized data; process of convergence on the basis of information technologies. The article contains the modernized content of course technology that realizes this concept.
\end{abstract}

\section{Introduction}

The convergence of information and material technologies has found its implementation in robotic engineering, while the convergence of information and cognitive technologies was realized in the knowledge gaining technology and learning skills.

The development of technology is closely related to the scientific knowledge. Moreover, the ultimate goal of science (for at least 400 recent years) has been namely the creation of technologies, while all together they were considered as acquisition of force and power (F. Bacon, T. Hobbes, etc.).

In XX century, the essence of technology was comprehended in various aspects:

- In the terms of mathematics, abstract structures were distinguished to be correlated to the informative concept of technology: concepts of algorithm and calculus (A. Church, A. Turing, E. Post, etc.), abstract structures of management (N. Wiener, A. N. Kolmogorov, etc.);

- Philosophy of technique and technological society in the whole (M. Heidegger, K. Jaspers, etc.);

- Social and civilization aspects of technology (M. Weber, W. Sombart, etc.).

\footnotetext{
* Corresponding author: srg57@mail.ru
} 


\section{Methods}

In the current world, NBICS technologies (nano-, bio-, information, cognitive, social technologies) are becoming the basis for realization of convergent solutions.

By the present time, all these aspects of technology have already overstepped the frames of special fields of knowledge and turned into a part of the current social context.

Due to the aforesaid reasons, the convergent technologies are becoming the object of study in the general educational disciplines of Informatics and Technologies, which are starting to interact with each other according to the principle of convergence.

\section{Results and discussion}

A fundamental objective of general education is to make the students learning the most significant aspects of reality. Comprehension of the information factor in early 1960s ultimately resulted in creation of the general educational course of informatics in 1985 .

At the present time, we can state that the course of informatics has been fully developed as a discipline in the cycle of natural sciences. The very science of informatics was considered there as a fundamental science, which studied the regularities of information processes taking part in the systems of various nature as well as by the methods, means and technologies of their automation.

At the same time, we can note also the fact that in the recent years the general educational course of informatics was placed in the artificial frames, which essentially restrict its potential capacities. In such restricted form it is far from answering the challenges of the current information civilization.

Today at least two such challenges can be clearly seen in the information sector:

- Comprehension of the "back side" of the information technology - in more conventional terms - "information security." There is no doubt that any means and any tool will have the limits of the own application and the own "contraindications." If no, sooner or later, questions will arise in relation to safety and, further, to expedience in application of similar means. Use of information technologies has already approached the line, where we should give the answers to these questions at the conceptual level rather than merely state the existence of such a problem.

- In the recent years, the old idea of interdisciplinarity has transformed into a qualitatively new concept of convergence, which is especially significant in application to technologies. The convergence of information and material technologies, information and cognitive technologies is not a notional construction but a strict need of practice. The convergence of information and material technologies has found the own implementation in robotic engineering, while the convergence information and cognitive technologies has found the own implementation in the knowledge gaining technology and learning skills.

In order to respond adequately to these challenges, it is necessary to expand the object basis of informatics, primarily in the technological aspect, to get beyond the very field of information technologies and to comprehend the phenomenon of technology as such, in the context of the human transforming activity.

The activity for purposeful transformation of the world around us exists for the same time as the humanity. However, this activity started to acquire the current features along with development of machined manufacture and related changes in the intellectual and practical human activities.

The conceptual aspect of these changes was clearly formulated by R. Descartes in his fundamental work Discours de la méthode.... According to the idea of Descartes, any activity should be carried out in compliance with some method, whereas the efficiency of this method directly depends upon the degree of its formalizability. This provision became 
the fundamental paradigm of the social structure, which is traditionally referred to as the "industrial society" and which was "inherited" by the information society.

The backbone of the aforesaid social structures is technology as the logical development of the Cartesian "method" in the following aspects:

- process for achievement of the set goal is formalized to such extent that it becomes possible to reproduce it in a wide range of conditions with the virtually identical results;

- there arises a principal opportunity for automation of the product manufacturing processes (that is gradually covering virtually all the aspects of human life).

The development of technology is closely associated with the scientific knowledge. Moreover, the ultimate goal of science (at least, for 400 recent years) is namely creation of technologies, while this was considered altogether as acquisition of force and power ( $\mathrm{F}$. Bacon, T. Hobbes, etc.).

Due to various reasons, informatics as the most mobile school disciplines failed to solve this problem. A probable way of such solution is seen in transformation of technology as another essential school discipline.

Such transformation was implemented by the working group, which included the authors of this article. The essence of their proposals was summarized as follows.

The principal objectives of a current course of technologies should be, in particular:

- determination of the personal and social needs characterizing the industrial and postindustrial (information) society, distinguishing the personal and social priorities;

- development, on the level of general education, of the methods and meals of the human teaching activity targeted to satisfaction of the formulated needs;

- forecasting the results, potential social and ecological consequences from the human transforming activity.

Upon completion of the course of technology, a student should have a formed platform of technological competence as a necessary component of his/her general culture and introduction of engineering culture

So, the unique variety of the human transforming activity excludes an opportunity of even a surface review of this activity in the terms of the school course of technology. On the other hand, the educational value of such a review is extremely low.

The traditional approach comprises selection of some conventional materials (paper, fabric, wood, metal, etc.), as well as some everyday works (repair of home wiring, agricultural works, etc.), which enable direct realization of the transforming activities of students. The course of these activities:

- forms the working skills, which are essential for life;

- gives the concept of transforming activity on the whole;

- provides intellectual development of students and promotes to educational process;

- carries out the process of professional orientation and pre-professional training.

For the certain period of time, such approach recommended itself as sufficiently effective. However, today this approach seems inadequate with respect to the specific features of the current information social environment and developed educational realities.

The most significant changes, which should be expressed in the course of technology, are as follows:

- Technologization in all the aspects of human activities is so great-scaled that the intuitive concepts of the essence and structure of technological process, which are formed by students leaving the secondary school, is far from being sufficient for their successful socialization.

- Development of the purely information and communication technologies resulted in the significant domination of the information sphere over the sphere of matter and energy that is certainly a negative phenomenon. Further development of the technological sphere is 
associated primarily with the convergence of material and information technologies implemented, in particular, in robotic engineering;

- One of the consequences from unprecedented development of the information sphere was a misbalance between the semantic and syntactic components of information. As a result, there emerged a situation, where the "wheel of casualty" among the data, information and knowledge fails to "turn." This indicates to the necessity in development of the principally new technologies, namely information and cognitive technologies targeted to the students acquiring knowledge and developing the learning skills.

Certainly, this new context does not reduce by any means but rather enhances the significance of handicrafts for formation of intellect and adequate concepts of the surrounding world.

In our opinion, the context formulated above requires different approaches to design of the contents and structure for the modern course in Technology.

It is expedient to make the central focus in the following points:

- Purposeful development in the essence of technology;

- Development of the methods for realization of the technological approach to solution of problems in any fields of human activities;

- Development of the skills of handicrafts, modeling, designing and projecting.

This assumes development of:

- General structure of technology as a combination of stages, operations and actions directed to achievement of the given goals or to creation of the products with the predetermined properties and parameters;

- Full-cycle structure for problem solution including the following stages: setting up a problem, selection or creation of the technology to be adequate to the given problem, realization of technology by means of the available assets and tools, estimation and correction of the obtained results and further use thereof.

It should be noted that namely the structural approach is the most correct and effective from the viewpoint of the current state of theoretical knowledge (Bourbaki program, "physical structures," etc.). Development of these structures is implemented in the course of:

- Handicrafts with traditional materials (paper, fabric, wood, metal);

- Designing models with application of a robotic construction kit;

- Solution of practically oriented problems;

- Realization of creative projects;

- Study of real technological processes in the spheres of matter, energy and information, in particular, by means of visual tools.

The key methodological tool for the general education course of technology is the robotic engineering complex, which enables to demonstrate the capacities of convergent technologies and to develop the skills of designing and projecting. On the basis of such robotic construction kit, we can not only design any models but also solve any practically oriented problems and to implement any creative projects.

The approximate distribution of academic hours among the aforementioned kinds of school activities may be as follows:

- Handicrafts - 20\%;

- Model designing with application of robotic construction kit - 30\%;

- Solution of practically oriented problems - 30\%;

- Implementation of creative e projects - $20 \%$.

On the whole, application of a robotic construction kit in the classes of technology may comprise up to $50 \%$ academic hours. 
The formulated approach was realized through a set of manuals for the course of technology in general school (years 5 to 8 ) by the team of authors (S. A. Beshenkov, V. B. Labutin, E. V. Mindzaeva, V. N. Ryagin, M. I. Shutikova), edited by S. A. Beshenkov, as prepared at the BINOM Publishing House in collaboration with the Lego Education Corporation.

\section{References}

1. S. A. Beshenkov, E. V. Mindzaeva, E. V. Beshenkova, M. I. Shutikova, I. I. Trubina, Smart education and e-learning (Springer, Cham, 2016)

2. I. V. Robert, I. S. Mukhametzyanov, A. A. Arinushkina, V. A. Kastornova, L. P. Martirosyan. Espacios, 38, 40 (2017)

3. I. Robert, L. Martirosyan, N. Gerova, V. Kastornova, I. Mukhametzyanov, A. Dimova, Smart education and e-learning (Springer, Cham, 2016)

4. S. Ryagin, I. Mukhametzyanov, A. Dimova, Mediterranean Journal of Social Sciences, 6, S5 (2015)

5. S. S. Neustroev, A. V. Simonov, Human and Education, 3, 44 (2015)

6. S. A. Beshenkov, E. V. Mindzayeva, Materials of cycles of the All-Russian teleconferences concerning federal state educational standards of the second generation: natural-science disciplines (BINOM: Laboratory of Knowledge, Moscow, 2009)

7. S. A. Beshenkov, M. I. Shutikova, E. V. Mindzaeva, The Teacher of XXI Century, 3 (2016)

8. S. A. Beshenkov, E. V. Mindzaeva, Distance and Virtual Learning, 5 (2016) 